\title{
Quadruple suspension design for Advanced LIGO
}

This article has been downloaded from IOPscience. Please scroll down to see the full text article. 2002 Class. Quantum Grav. 194043

(http://iopscience.iop.org/0264-9381/19/15/311)

View the table of contents for this issue, or go to the journal homepage for more

Download details:

IP Address: 194.94.224.254

The article was downloaded on 12/10/2010 at $13: 10$

Please note that terms and conditions apply. 


\title{
Quadruple suspension design for Advanced LIGO
}

\author{
N A Robertson ${ }^{1,5}$, G Cagnoli $^{1}$, D R M Crooks ${ }^{1}$, E Elliffe ${ }^{1}$, J E Faller ${ }^{2}$, \\ P Fritschel $^{3}$, S Goßler ${ }^{4}$, A Grant ${ }^{1}$, A Heptonstall ${ }^{1}$, J Hough ${ }^{1}$, H Lück ${ }^{4}$, \\ R Mittleman ${ }^{3}$, M Perreur-Lloyd ${ }^{1}$, M V Plissi ${ }^{1}$, S Rowan ${ }^{1,5}$, \\ D H Shoemaker ${ }^{3}$, P H Sneddon ${ }^{1}$, K A Strain ${ }^{1}$, C I Torrie ${ }^{1,6}$, H Ward $^{1}$ \\ and $\mathbf{P}$ Willems ${ }^{6}$ \\ ${ }^{1}$ Department of Physics and Astronomy, University of Glasgow, Glasgow G12 8QQ, UK \\ 2 JILA, NIST and University of Colorado, Boulder, CO 80309, USA \\ ${ }^{3}$ LIGO Laboratory, Massachusetts Institute of Technology, 175 Albany St, Cambridge, MA \\ 02139, USA \\ ${ }^{4}$ Universitat Hannover, Institut für Atom und Molekülphysik, Abteilung Spektroskopie, D30167, \\ Hannover, Germany \\ ${ }^{5}$ Department of Applied Physics, Ginzton Laboratory, Stanford University, Stanford, CA 94305, \\ USA \\ ${ }^{6}$ LIGO Laboratory, California Institute of Technology, MS 18-34, Pasadena, CA 91125, USA
}

Received 10 May 2002

Published 15 July 2002

Online at stacks.iop.org/CQG/19/4043

\begin{abstract}
In this paper, we describe the conceptual design for the suspension system for the test masses for Advanced LIGO, the planned upgrade to LIGO, the US laser interferometric gravitational-wave observatory. The design is based on the triple pendulum design developed for GEO 600 - the German/UK interferometric gravitational wave detector. The GEO design incorporates fused silica fibres of circular cross-section attached to the fused silica mirror (test mass) in the lowest pendulum stage, in order to minimize the thermal noise from the pendulum modes. The damping of the low-frequency modes of the triple pendulum is achieved by using co-located sensors and actuators at the highest mass of the triple pendulum. Another feature of the design is that global control forces acting on the mirrors, used to maintain the output of the interferometer on a dark fringe, are applied via a triple reaction pendulum, so that these forces can be implemented via a seismically isolated platform. These techniques have been extended to meet the more stringent noise levels planned for in Advanced LIGO. In particular, the Advanced LIGO baseline design requires a quadruple pendulum with a final stage consisting of a $40 \mathrm{~kg}$ sapphire mirror, suspended on fused silica ribbons or fibres. The design is chosen to aim to reach a target noise contribution from the suspension corresponding to a displacement sensitivity of $10^{-19} \mathrm{~m} \mathrm{~Hz}^{-1 / 2}$ at $10 \mathrm{~Hz}$ at each of the test masses.
\end{abstract}

PACS number: $0480 \mathrm{~N}$ 


\section{Introduction}

The sensitivity of the interferometric gravitational wave detector presently installed in the US LIGO [1] is expected to be limited by the thermal noise associated with the suspensions of its mirrors at frequencies in the region $\sim 40 \mathrm{~Hz}$ to $\sim 150 \mathrm{~Hz}$. The LIGO suspension design $[2,3]$ for the main mirrors has the following features.

- The fused silica mirrors $(10.7 \mathrm{~kg})$ are hung as single pendulums on a single loop of steel piano wire.

- The sensing and actuation for damping of the low-frequency pendulum modes are carried out at the mirror itself, with the magnets for actuation attached to the back and side of the mirrors via metal standoffs.

- Actuation for global control, required to hold the interferometer at its correct operating position, is also carried out via the magnets attached to the mirrors.

In GEO 600 [4], the German/UK interferometric gravitational wave detector, the approach to the suspension system represents a second-generation design for which the performance is more aggressive than in LIGO, in particular in terms of the reduction of thermal noise associated with the suspension of the mirrors. The GEO design incorporates fused silica fibres of circular cross-section to suspend the fused silica mirror in the lowest stage of a triple pendulum, the damping of whose low-frequency modes is achieved by using co-located sensors and actuators at the highest mass of the triple pendulum. Global control forces are applied via a triple reaction pendulum, so that these forces can be implemented from a seismically isolated platform. These design features have been discussed in previous papers [5-10]. Figure 1 shows a schematic diagram of the GEO suspension system and a picture of the first triple pendulum to be assembled with a monolithic fused silica final stage, hanging in situ in one of the GEO tanks.

The more advanced suspension design has been used in GEO to compensate for its shorter arm length (600 m compared to $4 \mathrm{~km}$ ), in order to achieve a similar strain sensitivity to LIGO. Operating these detectors at their design sensitivities will be an exciting step forward in the quest for detecting gravitational waves, and may lead to their first detection. However, to realize the possibility of carrying out serious astronomy using gravitational waves, further improvement in sensitivity is required. An obvious step is to adapt the more advanced suspension design of GEO in the planned upgrade to LIGO, and this has been proposed in the white paper [11] put forward by the LIGO Scientific Collaboration to the National Science Foundation describing the next generation of LIGO. The GEO team, in collaboration with LIGO and other members of the LIGO Scientific Collaboration, has been developing the suspension design to meet the requirements for Advanced LIGO. In particular, we are designing a quadruple pendulum suspension for the main mirrors, which is an extension of the GEO design. The key features of the proposed design are as follows.

- Sapphire mirrors (40 kg) will form the lowest stage of a quadruple pendulum, and will be suspended on four vertical fused silica fibres or ribbons to reduce suspension thermal noise.

- The fibres will be welded to fused silica 'ears' or prisms which are silicate bonded [8] to the flat sides of the penultimate mass and the mirror below. This technique ensures that the low mechanical loss of the mirror itself is preserved, maintaining the low thermal noise of the sapphire substrate.

- Included in the quadruple pendulum are three stages of cantilever blade springs made of maraging steel to enhance the vertical seismic isolation. 


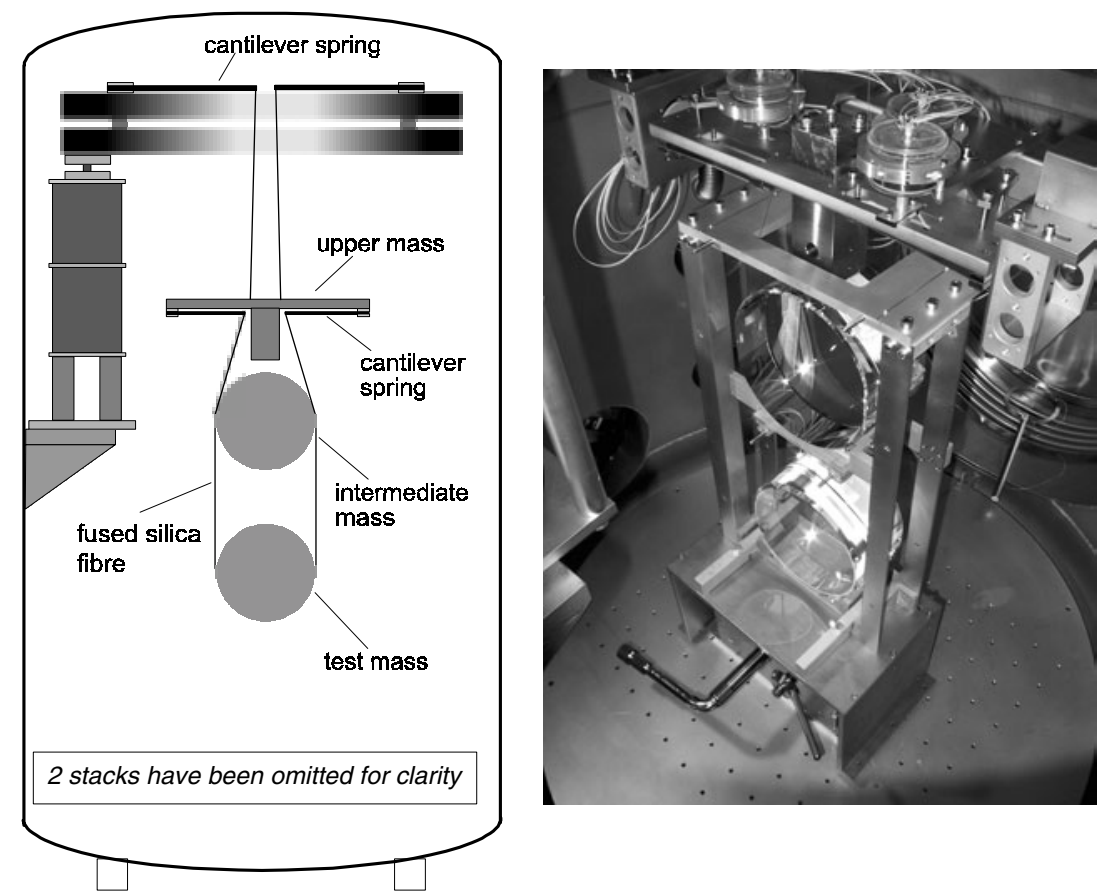

Figure 1. Schematic diagram (left) of the full suspension and isolation system for the main mirrors (test masses) in GEO 600, and a picture of the first triple pendulum with monolithic final stage hanging in situ in one of the GEO tanks. Three of the coil actuators for local control can be seen above the upper mass of the triple pendulum.

- The damping of all of the low-frequency modes of the quadruple pendulum will be carried out either by using six co-located sensors and actuators at the highest mass of the pendulum (as in GEO), or by using eddy current damping applied at this mass. To achieve adequate damping the design has to be such that all the modes couple well to motion of the highest mass.

- DC alignment of mirror yaw and pitch will be done by applying forces to the actuators at the highest mass, or at the mass below. The masses hanging below the highest mass are each suspended by four wires, two on each side, so that the system behaves like a marionette from the highest mass downwards.

- Global longitudinal and angular control forces will be applied via a reaction pendulum, essentially identical in mechanical design to the main pendulum, but with wires replacing the silica fibres.

- Global control will be carried out using a hierarchical feedback system, with large low-frequency forces applied electromagnetically between the penultimate masses, and small higher-frequency signals applied electrostatically between the mirror and the corresponding lowest reaction mass which may be made of silica or heavy glass with a patterned gold coating. Alternatively, photon pressure from an auxiliary laser may be used for the higher-frequency signals, in which case the lowest reaction mass is not required.

The extension from a triple pendulum as in GEO 600 to a quadruple pendulum for Advanced LIGO is necessary to meet the more stringent requirements on isolation of noise associated with the damping of the low-frequency pendulum modes, discussed in section 3.2. 

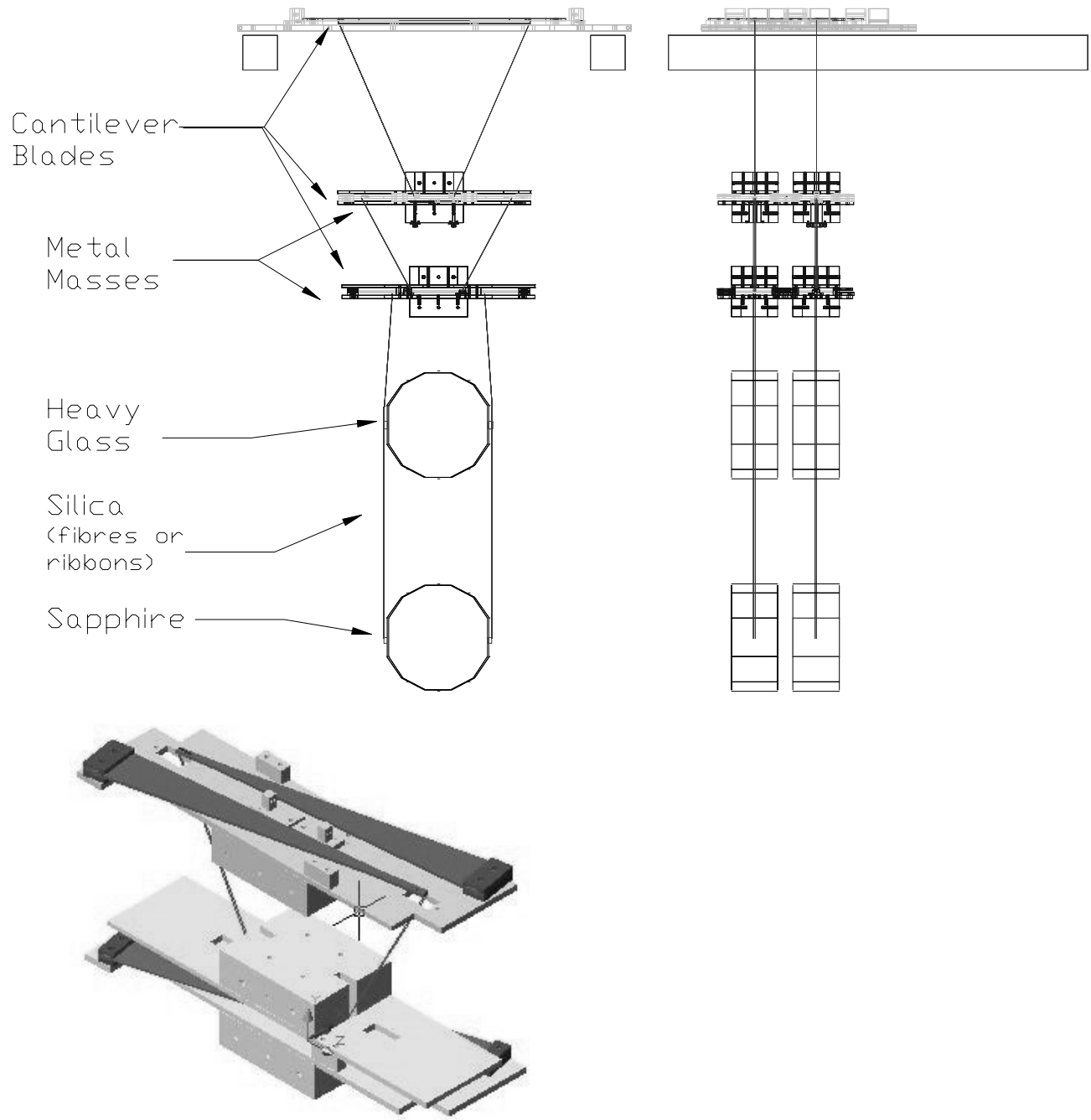

Figure 2. Schematic diagram of quadruple pendulum suspension system for Advanced LIGO. The diagram above shows a face view of the main chain on the left, and on the right a side view with main and reaction chains is visible. The diagram below shows a close up of the first two masses (masses 1 and 2), with the top of mass 1 removed so that the cantilever blades for vertical isolation, which are crossed to save space, can be seen more clearly.

Figure 2 shows a schematic diagram of our present conceptual design for the quadruple pendulum suspension. We discuss the features of the proposed design in more detail below, addressing the various issues, and giving predictions of the performance of the suspension system.

\section{Thermal noise issues}

\subsection{Some general considerations}

Thermal noise, or motion due to the thermal energy, sets a fundamental limit to the noise performance of the suspension, and is thus the paramount design driver. The main contribution 
from the suspension per se comes from the dissipation in the fused silica fibres used to suspend the mirror, giving a direct optical axis noise component. To minimize this noise, the baseline design currently incorporates ribbons rather than fibres of circular cross-section, so that the dilution factor [12], by which the pendulum loss factor is reduced from the value of the intrinsic loss factor of the suspension material, is increased. The choice between ribbon and cylindrical fibre is discussed more fully below; we will refer to both as 'fibres' when the distinction is not needed.

Another strong contributor to the thermal noise spectrum arises from the lowest set of blade springs, giving a vertical noise component which will cross-couple into horizontal motion. In general, thermal noise arising further up the pendulum chain is filtered by the stages below. However, the vertical frequency of the final stage is necessarily higher than the horizontal frequency, since no blades are included at that stage, and thus there is less vertical filtering.

Since it is desirable from astrophysical arguments to extend the working frequency of the detector downwards as far as is experimentally practicable, we are considering a baseline design for Advanced LIGO which has a 'cut-off' in the vicinity of $10 \mathrm{~Hz}$. Below this frequency the noise will rise steeply to lower frequencies due to seismic effects, essentially giving a cutoff in detector sensitivity. Our working requirement is that the required noise level at each of the test mirrors be $10^{-19} \mathrm{~m} \mathrm{~Hz}^{-1 / 2}$ at $10 \mathrm{~Hz}$, falling off at higher frequencies. To achieve such a requirement calls for the highest vertical mode of the multiple pendulum to be kept below $10 \mathrm{~Hz}$. The highest mode essentially corresponds to relative vertical motion of the mirror with respect to the penultimate mass. To push this frequency down, we use a combination of several factors:

(a) The fibre length is chosen as long as practicable, consistent with ease of production and the need to maintain the 'violin' modes high enough for control purposes. The current design target is $60 \mathrm{~cm}$.

(b) The fibre cross-section is chosen to be as small as practicable, consistent with working at least a factor of 3 away from the breaking stress.

(c) The penultimate mass is chosen to be as heavy as possible, consistent with the overall design characteristics of the multiple pendulum. In the baseline design we have chosen to make this mass approximately double the mass of the mirror.

To achieve a penultimate mass which can be bonded, we are considering the use of heavy glass (glass doped with lead or other dense metals).

We will return to these design factors after consideration of the choice of ribbons or cylindrical fibres.

\subsection{Ribbons and fibres}

There are potential advantages to using ribbons rather than cylindrical fibres, and these have already been discussed elsewhere [13-15]. Not only can the dilution factor be made larger for ribbons, but reducing the thickness of the flexing element also raises the frequency at which the maximum loss due to thermoelastic damping occurs [16], which can lead to a lower overall level of noise around $10 \mathrm{~Hz}$. Experimental results on losses in ribbons have also been carried out [17], and these are encouraging. However, there are several other factors which need to be considered before a choice can be made.

Firstly, recent work by Cagnoli and Willems [18] has shown that there is a significant thermoelastic effect not previously considered, basically due to the variation of Young's modulus with temperature. This effect, in combination with the more familiar coefficient of 
thermal expansion, gives rise to an effective coefficient of thermal expansion which can be zero for a particular static stress. Hence, under those conditions the thermoelastic damping can become arbitrarily small, and also the overall noise level is reduced. The null condition can, in principle, be achieved by increasing the cross-section of the silica suspension over that which has been previously indicated as optimum from other design considerations. However, simply increasing the cross-section to null the thermoelastic effect has the two adverse consequences of increasing the highest vertical pendulum mode above the $10 \mathrm{~Hz}$ goal, and of decreasing the violin mode frequencies, thus placing more of these resonances below $1 \mathrm{kHz}$ and complicating the control design.

An alternative possibility which has recently been suggested [19] is to use circular crosssection fibres of varying cross-section, thicker near the ends and thinner in the middle section, such that the thermoelastic effect is reduced, but also that the highest vertical mode is kept below $10 \mathrm{~Hz}$. Similar tailoring of ribbons could also yield enhanced performance. These ideas are being pursued for possible incorporation into the design.

A second consideration is the breaking stress of ribbons and cylindrical fibres, and the ease with which they can be made. Measurements on cylindrical fibres have shown that they can be as strong as high tensile steel $[20,10]$, and we now achieve an average value of breaking stress of $\sim 4.5 \mathrm{GPa}$. Ribbons with breaking stress comparable to the strongest fibres have yet to be developed. However, this is an active area of research, and initial results at Glasgow have already shown breaking stresses in excess of $1.8 \mathrm{GPa}$.

An additional complication with ribbons is the need to allow flexing without buckling in both the plane, and perpendicular to the plane, of the ribbon. Twists or other flexures may be needed. Again, this is an area of research.

In conclusion, it can be seen that there are various issues in the suspension design which as yet are unresolved. The final design choice of ribbons or cylindrical fibres, possibly with varying cross-section, will depend on the results of investigations of such matters as reliability of manufacture, strength and loss measurements, and controls design. For the purposes of this baseline design we use ribbons of constant cross-section for our estimation of expected thermal noise in a quadruple suspension system.

\subsection{Thermal noise estimation for quadruple pendulum suspension}

The thermal noise model which has been used for this estimation has been developed using MAPLE. It has subsequently been modified into MATLAB code for inclusion in the BENCH modelling tool (http://gravity.phys.psu.edu/Bench) which has been developed as a tool for predicting the astrophysical range for various potential sources, for varying parameters of detector configuration for Advanced LIGO. Some details of how the thermal noise calculations are carried out are presented in appendix A1. Examples of pendulum thermal noise spectra produced using the MAPLE code are given in section 4.

\section{Isolation, damping and control}

Modelling for investigation and optimization of the mechanical design for a quadruple suspension, with particular reference to the isolation and damping properties, has been carried out using an extension of the MATLAB model developed for the GEO 600 triple suspension $[5,21]$. Some details of the MATLAB model are presented in appendix A2.

The key elements of the design are very similar to GEO, with the addition of another stage. The aim has once again been to develop a model whose coupled resonant frequencies all lie within a band from $\sim 0.4$ to $\sim 4 \mathrm{~Hz}$, with the exception of the highest vertical and roll 
modes which are associated with the extension of the silica fibres in the lowest pendulum stage. In addition, we aim for good coupling of all the low-frequency modes, so that damping of all such modes can be carried out at the top mass in the chain.

\subsection{Mechanical design}

The mass at the top is suspended from two cantilever-mounted, approximately trapezoidal pre-curved spring blades and two spring steel wires. The blades are made from Marval $18(18 \% \mathrm{Ni})$ maraging (precipitation hardened) steel, chosen for its high tensile strength and low creep under stress, as used in the French-Italian VIRGO gravitational wave detector project [22]. The blades lie horizontally when loaded. The mass below this is suspended from two cantilever blades and two steel wire loops. The top mass (mass 1) and mass 2 have a 'sandwich-type' construction with the blades fitting in between, so that the break-off points for wires going both upwards and downwards lie close to the centre of mass of these masses; see figure 2. Mass 3, which may be made of heavy glass, is suspended from two cantilever blades and two steel wire loops from mass 2. Fused silica ears silicate bonded to flats on the side of this mass form the fibre attachment points at the mass. Similar ears are bonded to the mirror (mass 4), and the final suspension is made by welding cylindrical fibres or ribbons between the ears of masses 3 and 4, with two fibres on each side.

There are several key points which differ from the original GEO design. Firstly, in order to achieve a smaller footprint, all the blades are angled with respect to each other and crossed (as shown in figure 2). In GEO only the top set of blades in the beamsplitter suspension were crossed. Secondly, again due to space considerations, there are two blades rather than four at masses 1 and 2, each blade supporting two wires from its end. As stated earlier, the overall choice of the number of wires or fibres is such that orientation of the mirror can be carried out from the top mass.

Currently, we have chosen to stress the blades at a conservative level, to approximately one half of the elastic limit $(\sim 800 \mathrm{MPa})$ for the blades closest to the mirror and slightly larger (up to $\sim 900 \mathrm{MPa}$ ) for those further from the mirror. However, we may choose to increase the stress slightly to raise the internal mode frequencies of the blades, as discussed in section 5.

There should be a strong coupling of all degrees of freedom to motion of sensors/actuators at the top mass. To a first approximation, this is satisfied by having approximately the same mass in each stage, approximately the same moments of inertia about equivalent axes, and by suitable choices of wire angles and connection points. In this particular design, thermal noise considerations have necessitated the use of a significantly heavier penultimate mass than the other masses in the chain.

\subsection{Local control}

In GEO, the active local control damping is applied at the top mass ensuring that the pendulum stages below filter any extra motion caused by electronic noise in the feedback system. However, given the more ambitious target noise level for the LIGO suspensions of $10^{-19} \mathrm{~m} \mathrm{~Hz}^{-1 / 2}$ at $10 \mathrm{~Hz}$, the GEO design needs some modification. In particular, to provide more isolation from the noise associated with the local damping, the suspension is increased from three to four stages, with local damping still applied only to the top mass. Even then, local sensing noise can dominate. Typical optical shadow sensors $[23,24]$ with a range of $\sim 1 \mathrm{~mm}$ have a noise level of $\sim 10^{-10} \mathrm{~m} \mathrm{~Hz}^{-1 / 2}$, much greater than the $\sim 10^{-19} \mathrm{~m} \mathrm{~Hz}^{-1 / 2}$ target for the suspensions at $10 \mathrm{~Hz}$. However, the mechanical isolation from the sensed point to the test mass is only of order $10^{-7}$ (see figure 7) at $10 \mathrm{~Hz}$. Thus, the sensor noise-isolation product 
is greater than the target sensitivity of the target by at least two orders of magnitude. In GEO, there is roughly a decade between the highest locally damped suspension mode and the $50 \mathrm{~Hz}$ lower edge of the sensitive frequency band-enough room to electronically filter local sensor noise to a level below the target sensitivity. At Advanced LIGO's $10 \mathrm{~Hz}$ cut-off frequency, however, little electronic filtering can be achieved while maintaining adequate phase margin in the damping loops.

A partial solution to the local sensing noise problem is provided by the interferometer global sensing system [25]. In the power-recycled, Fabry-Perot arm Michelson interferometer configuration, four interferometric relative position signals are generated by the relative longitudinal movements of the test masses, beamsplitter and power recycling mirror; with the addition of signal recycling in Advanced LIGO, one mirror is added and thus one further interferometric position signal is obtained. These interferometric position signals all have sensitivities better than $10^{-13} \mathrm{~m} \mathrm{~Hz}^{-1 / 2}$, i.e., at least three orders of magnitude better than the local shadow sensors. Thus we can use four of these global signals to control the longitudinal degrees of freedom of the four test masses. When this is done, the local longitudinal damping of the test masses can be greatly reduced, or even turned off, to suppress local sensor noise. Similarly, low-noise global interferometric signals are available for the pitch and yaw orientation degrees of freedom of the test masses which can be used to control their pitch and yaw modes. The same mechanical coupling between the suspension stages that enables local damping forces applied at the top mass to effectively damp test mass motion, also allows that globally sensed motion of the test mass can be damped by actuation at the upper stages.

This scheme applies to all but the vertical, transverse and roll modes of suspension, which are not independently sensed by the interferometer. For these modes we could use one or more of several strategies to limit local damping noise: reduce the mechanical coupling from these degrees of freedom to the motion sensed by the gravitational wave readout; operate with reduced active damping, allowing higher $Q$ for these modes; take advantage of what limited electronic filtering can be performed on the local damping signals; or develop lower noise local sensors.

Eddy current damping may provide an alternative solution to active local control. Such damping is used in the Japanese TAMA project to damp their double pendulum suspensions [26]. In Advanced LIGO, we could use eddy current damping in six degrees of freedom applied at the top mass of the quadruple suspensions to give $Q$ of approximately 10 for the lowest frequency modes (which dominate the impulse response). We have estimated that residual motion at the mirror due to the thermal noise force generated by such eddy current damping is approximately $4 \times 10^{-20} \mathrm{~m} \mathrm{~Hz}^{-1 / 2}$ at $10 \mathrm{~Hz}$, which meets the target sensitivity. The final decision on how to apply damping will be made once more experimental investigations have been carried out.

\subsection{Global control}

The GEO philosophy for applying the feedback signals to the test masses for longitudinal and angular global control was briefly described in the introduction. The general idea is to apply forces between the main pendulum chain and an essentially identical reaction chain (which does not include fibre suspensions). The reaction chain is itself locally damped in the same manner as the main chain. In LIGO, however, not all the sensitive optics require wide bandwidth global control, and in those cases the reaction chain need not have as many stages. In addition, where wide bandwidth is required, the final stage wide-bandwidth smallsignal feedback could be realized using photon pressure from an auxiliary laser, rather than 
electrostatically as in GEO. In that case also the lowest stage of reaction chain would not be required.

Another issue is the potential need to damp (actively or passively) the very high $Q$ violin modes of the silica suspensions to allow the global feedback to remain stable. Any such damping has to be done in such a way as not to compromise the low-frequency thermal noise performance of the suspensions. In GEO we have taken the approach of using small amounts of amorphous PTFE coating on the fibres, suitably placed to damp the first few violin modes to $Q$ of around $10^{6}$, without compromising the low-frequency suspension noise. For GEO we use two coated regions each $5 \mathrm{~mm}$ long, one at the centre and one one-third of the way down the fibre. The LIGO situation has to be considered fully once a control philosophy has been decided upon, and there will be some trade-off required between controllability and thermal noise associated both with the low-frequency vertical modes and the violin modes.

\section{Expected performance}

In this section, we present various graphs, showing expected overall thermal noise performance, horizontal and vertical isolation performance with and without damping, and transfer functions from which residual sensor noise may be estimated. Key parameters used in the models to generate these graphs are also given. In some cases several curves are given, where different choices of parameters are possible.

\subsection{Key parameters}

The key parameters used for all the curves presented in this section are as follows (except where otherwise indicated):

\begin{tabular}{ll}
\hline Final mass & $40 \mathrm{~kg}$ sapphire, $31.4 \mathrm{~cm} \times 13 \mathrm{~cm}$ \\
Penultimate mass & $72 \mathrm{~kg}$ (heavy glass) \\
Upper masses & $36 \mathrm{~kg}, 36 \mathrm{~kg}$ \\
Overall length & $1.7 \mathrm{~m}$ (from top blade to centre of mirror) \\
Ribbon parameters & Length $60 \mathrm{~cm}$, cross-section $113 \mu \mathrm{m} \times 1.13 \mathrm{~mm}$ \\
Stress in ribbon & $770 \mathrm{MPa}$ \\
\hline
\end{tabular}

We note that in carrying out these analyses, the availability of sapphire pieces of the desired quality with these dimensions, and the availability of heavy glass of suitable density in the required size, are still open questions.

\subsection{Thermal noise performance}

In figure 3, we present the thermal noise for the baseline design. The target figure of $10^{-19} \mathrm{~m} \mathrm{~Hz}^{-1 / 2}$ at $10 \mathrm{~Hz}$ is effectively met. We also indicate the performance if the penultimate mass is made of silica rather than heavy glass, raising the uppermost vertical mode frequency of the quadruple pendulum to above $10 \mathrm{~Hz}$. Note that for the latter case, the blade designs were altered to keep the other three vertical resonant frequencies at the same values.

Various changes could be made to the baseline design. A marginal improvement to the performance at $10 \mathrm{~Hz}$ and above could be made if one lengthened the final stage to say $70 \mathrm{~cm}$. Increasing the cross-section of the fibre could gain some improvement above $10 \mathrm{~Hz}$ at the expense of raising the vertical resonant frequency to be closer to $10 \mathrm{~Hz}$, and lowering the violin mode frequencies. This improvement arises since changing the cross-section changes 


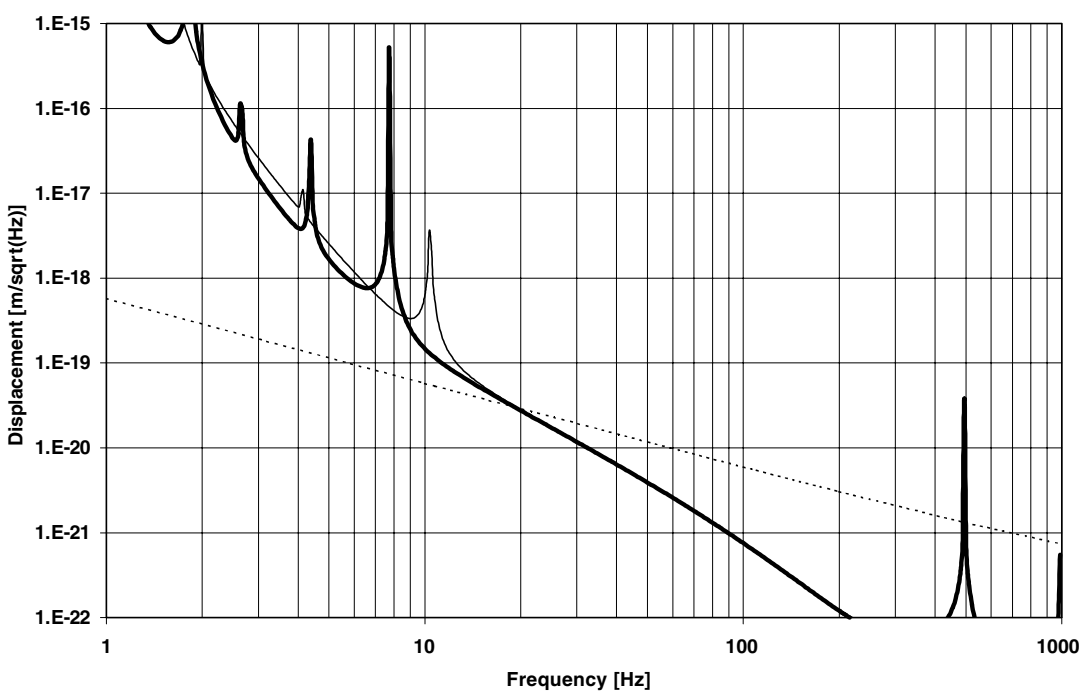

Figure 3. Suspension thermal noise for baseline $40 \mathrm{~kg}$ quadruple pendulum. Two suspension curves are shown. The heavy solid line is the baseline design. The light solid line shows the effect of replacing the $72 \mathrm{~kg}$ heavy glass penultimate mass with a silica mass of the same dimensions (weighing $22.1 \mathrm{~kg}$ ). The peaks of the resonances are not resolved. Note the first violin mode at approximately $500 \mathrm{~Hz}$. For comparison we also show the expected internal thermal noise curve for sapphire, dominated by thermoelastic damping (dotted line). Note also that the internal thermal noise curve assumes no loss due to coatings, or due to bonding of ears for attaching the suspensions.

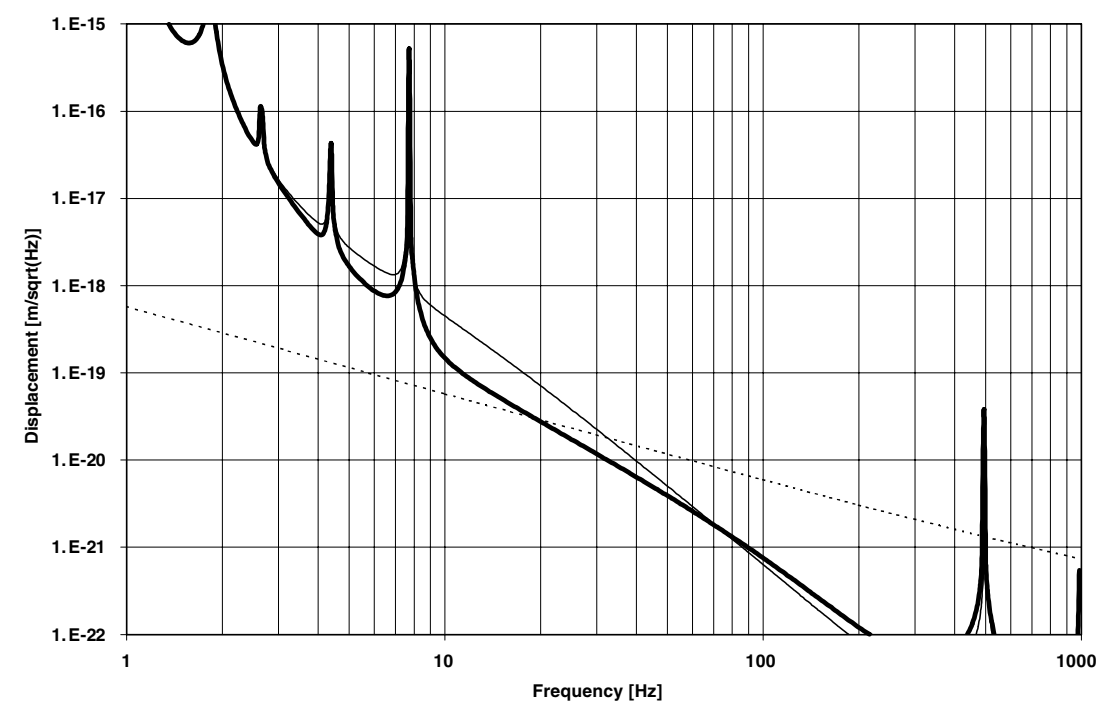

Figure 4. The light solid line is thermal noise for fibres of $200 \mu \mathrm{m}$ radius, stressed to the same value as the baseline ribbon design. The heavy solid line is the baseline, the dotted line is internal thermal noise for sapphire.

the position of the thermoelastic peak. Using cylindrical fibres loaded to the same stress as the baseline design (thus keeping the vertical mode frequency at the same value) raises the thermal noise in the $10 \mathrm{~Hz}$ region and above-as can be seen from figure 4 . 


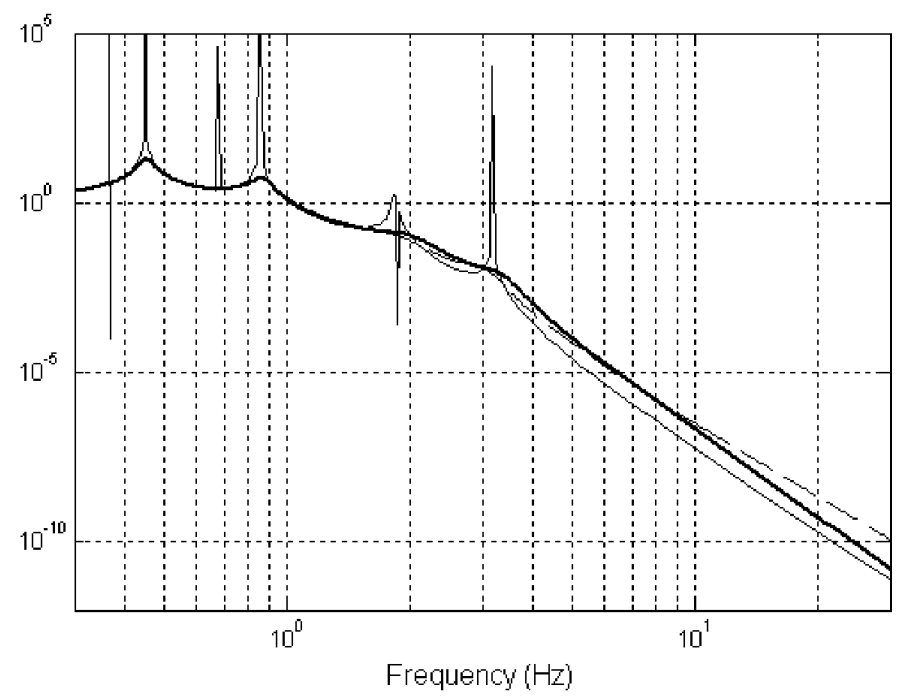

Figure 5. Longitudinal transfer function for quadruple pendulum, with (heavy solid line) and without (light solid line) local controls on, and with eddy current damping (dashed line).

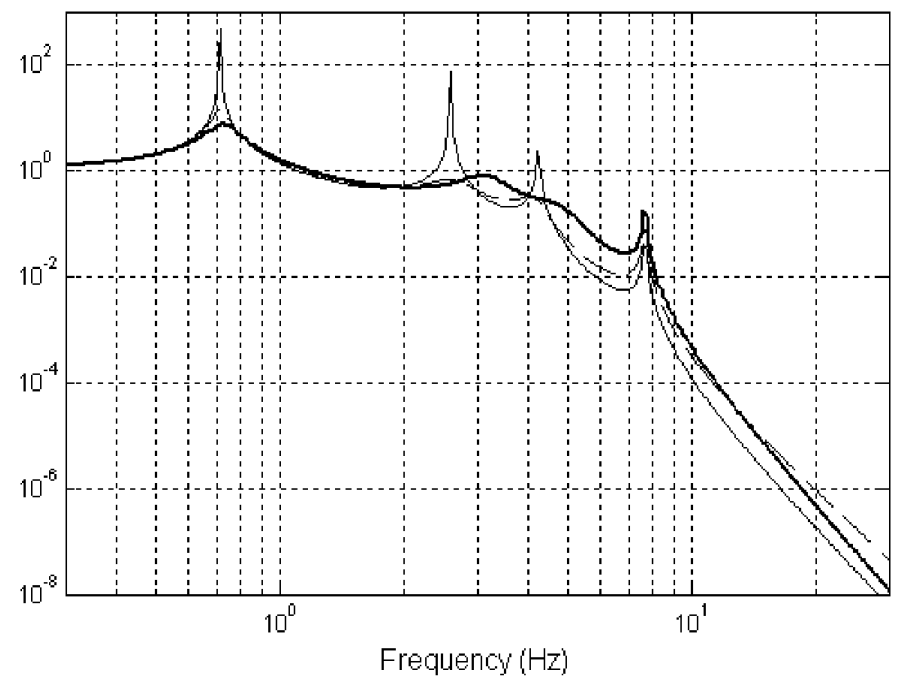

Figure 6. Vertical transfer function for quad pendulum, with (heavy solid line) and without (light solid line) local controls on, and with eddy current damping (dashed line).

\subsection{Isolation performance}

The overall mechanical isolation in Advanced LIGO will be achieved by a combination of a two-stage active isolation system [27] and the isolation from the quadruple suspension. The target noise level for the active system is $2 \times 10^{-13} \mathrm{~m} \mathrm{~Hz}^{-1 / 2}$ at $10 \mathrm{~Hz}$ in both longitudinal and vertical directions (where longitudinal refers to the horizontal direction along the beam axis). Figures 5 and 6 show the transfer functions for the quadruple pendulum in longitudinal and vertical directions respectively, from which the expected isolation performance can be deduced. The quadruple suspension has an isolation factor (with active or eddy current damping) 

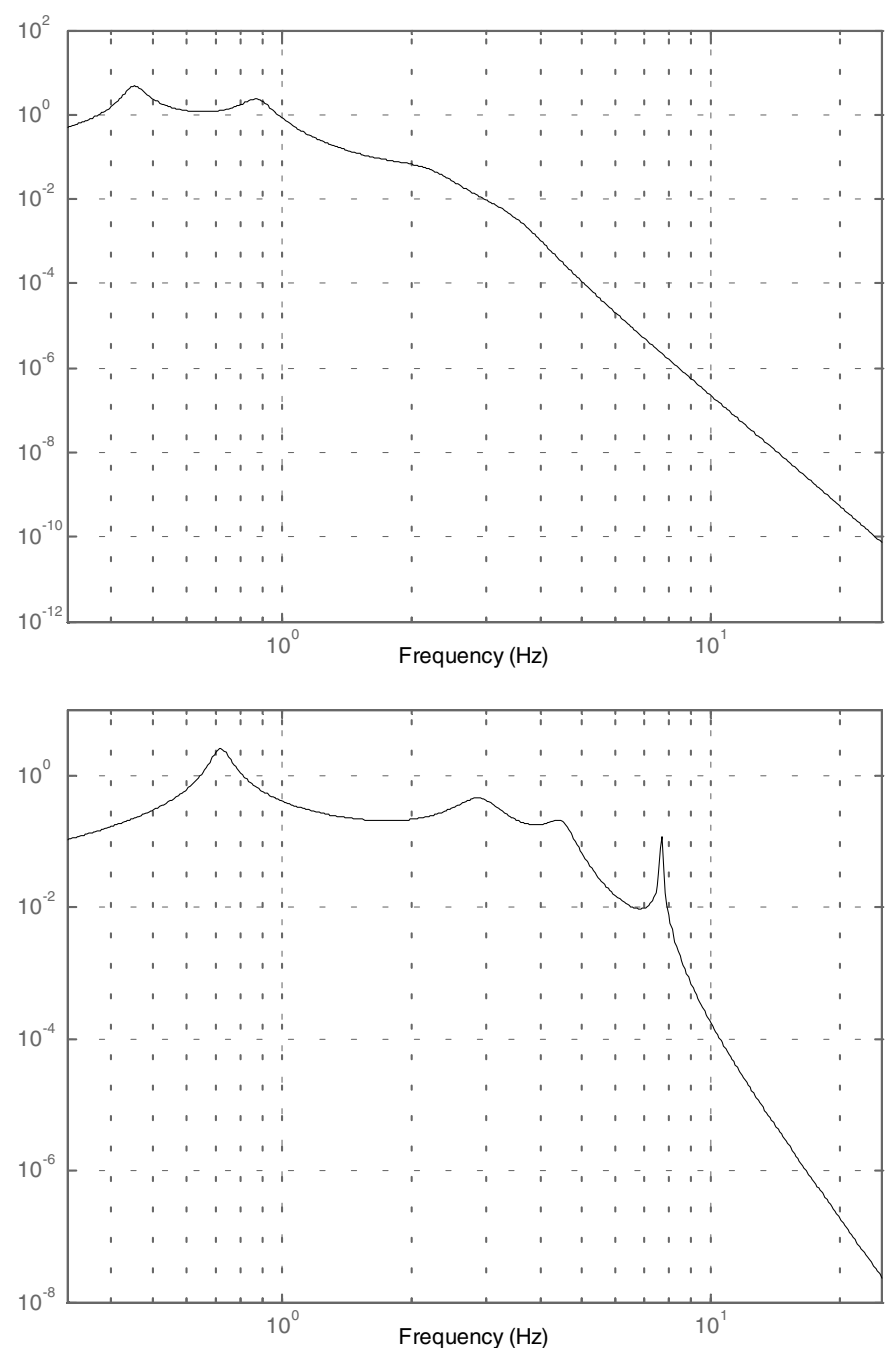

Figure 7. Longitudinal (left) and vertical transfer function from sensor to mirror for quadruple pendulum with sensor at the top mass.

of $\sim 4 \times 10^{-7}$ in the longitudinal dimension and $\sim 4 \times 10^{-4}$ in the vertical dimension at $10 \mathrm{~Hz}$. When these numbers are combined with the target noise level including a cross-coupling factor of $10^{-3}$ from the vertical to the horizontal dimension (see appendix A1), we see that the target sensitivity level of $10^{-19} \mathrm{~m} \mathrm{~Hz}^{-1 / 2}$ is achieved for both dimensions. The two-stage active isolation system also significantly attenuates the motion in the control band from 0.1 to $10 \mathrm{~Hz}$, reducing the actuator authority requirement in the suspension design.

\subsection{Sensor noise performance}

In figure 7 , we show the transfer function from the sensors to the mirror in both longitudinal and vertical directions using a nominal damping system gain which gives quality factors for the pendulum resonances of $\sim 10$ or less and corresponding impulse response times of $\sim 10 \mathrm{~s}$. The noise level at the mirror can be calculated from the transfer function shown in figure 7 
multiplied by the sensor noise in $\mathrm{m} \mathrm{Hz}^{-1 / 2}$. The longitudinal transfer function is $\sim 2 \times 10^{-7}$ at $10 \mathrm{~Hz}$. Thus, with a sensor noise of $10^{-10} \mathrm{~m} \mathrm{~Hz}^{-1 / 2}$ and no further electronic filtering the noise level at the test mass would be $\sim 2 \times 10^{-17} \mathrm{~m} \mathrm{~Hz}^{-1 / 2}$ at $10 \mathrm{~Hz}$, much larger than the target sensitivity. As discussed in section 3.2, a solution to this problem is to turn the gain down or off completely for the longitudinal modes once the global control of the interferometer is in operation and suitable signals from that control can be used to take over the damping.

For the vertical direction, the longitudinal noise level at the mirror can be calculated as above, with an extra factor, the cross-coupling factor, in the product. The vertical transfer function at $10 \mathrm{~Hz}$ is $\sim 2 \times 10^{-4}$, so with a sensor level of $10^{-10} \mathrm{~m} \mathrm{~Hz}^{-1 / 2}$, and assuming a cross-coupling factor of $10^{-3}$, the residual noise level at the mirror would be $\sim 2 \times 10^{-17} \mathrm{~m} \mathrm{~Hz}^{-1 / 2}$ at $10 \mathrm{~Hz}$, again far exceeding the target sensitivity. As discussed in section 3.2 , there are several strategies which could be used to address this issue, separately or in combination. With respect to the idea of turning down the gain once the global control is in operation, giving higher $Q$ for these modes, a more complete overall interferometer control model will be needed before it can be determined if the resulting larger motion could be tolerated.

\section{Current and future work}

Work towards developing a quadruple pendulum suspension as described above is well underway. Experience is being gained at GEO 600 with constructing and operating triple pendulum suspensions. This should give us information on many of the key aspects of the design, including thermal, isolation and damping properties and operation of global control.

To address thermal noise issues, ribbon and fibre production, including strength, reliability, welding and loss tests are being carried out in Glasgow and at Caltech. Investigation of bonding continues at Glasgow, Stanford and Caltech, with regard to bonding silica ears to sapphire and to lead or bismuth loaded glass, the latter materials being considered for the penultimate mass in the quadruple chain.

To address mechanical design, a first all metal prototype quadruple pendulum and reaction mass was developed in Glasgow early in 2001; parts were procured and shipped to MIT where they were assembled during summer 2001. Figure 8 shows pictures of the two quadruple pendulums (main chain and reaction chain), hanging in the lab at MIT in the summer of 2001. This suspension mimics a $30 \mathrm{~kg}$ sapphire mirror with an identically sized silica penultimate mass, which was a previous baseline design, now superceded with the design as discussed above. This prototype has already given us experience in assembly and handling. Current and future work includes measuring mode frequencies, and investigating transfer functions, damping and global control.

More work on blade design is underway, involving finite element analysis and comparison to experimental results. Another issue being considered is the noise level from the blades when thermally or seismically excited at their internal mode frequencies (in particular, the lowest set of blades nearest to the test masses). It is desired that the peaks at these frequencies not compromise the sensitivity, and damping may be needed to ensure this. For the design presented in section 4 the lowest internal modes were in the range 75 to $120 \mathrm{~Hz}$. Initial calculations suggest damping could be avoided if the frequencies are a little higher than these. Suitable frequencies could be achieved by allowing the maximum stress to be around $1050 \mathrm{MPa}$.

It should be noted that we have addressed the design of the most sensitive mirrors in Advanced LIGO in this paper, namely the end mirrors in the two cavities. However, the tools developed for designing the quadruple suspension can be easily applied for the design of other 

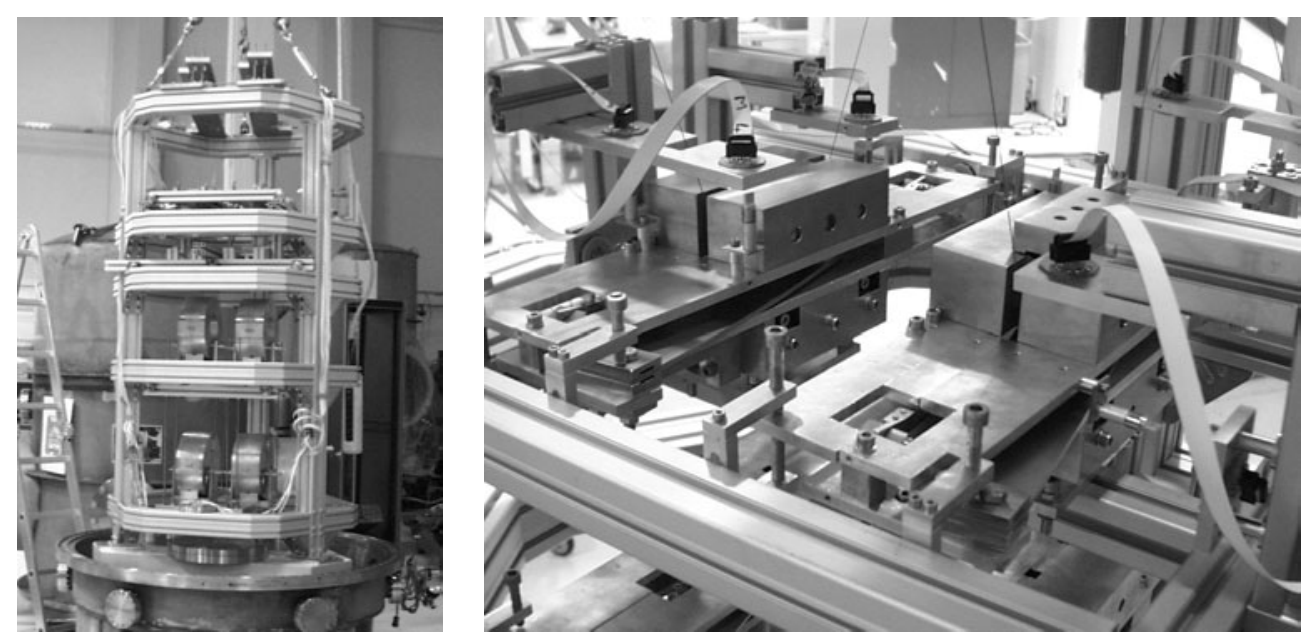

Figure 8. Two views of the prototype quadruple suspension assembled at MIT. On the left is an overall view showing the main and reaction chains, suspended from a support frame. On the right is a close-up of the top masses, with some of the local control actuators visible. The construction can be compared to the diagrams in figure 2 .

suspensions. In addition to the design issues mentioned above which are under investigation, there are several key issues still unresolved for the suspension design, some of which depend on other areas of research for Advanced LIGO. For example, the choice of mirror material and its size and aspect ratio are not yet fixed. Sapphire is presently favoured, and work is underway on investigating the growth of large enough pieces and investigating the optical properties such as absorption, inhomogeneity, polishing, etc. The fallback position is to use silica. Another area currently under discussion is the choice of the lower limit to the observation frequency for the Advanced LIGO instrument, and this has a bearing on the final design.

In conclusion, we have presented the current conceptual design of the suspension system for Advanced LIGO, which is based on the GEO suspension system. Experience with GEO will be invaluable as a test of the ideas incorporated in this design. However, much work has still to be carried out, and is actively underway in several laboratories in Europe and the USA.

\section{Acknowledgments}

The authors would like to thank their colleagues in the GEO collaboration for their interest and help in this work. We also acknowledge with thanks members of the LIGO Scientific Collaboration (LSC) at Caltech, MIT and Stanford who have contributed, in particular Mark Barton at Caltech. The Glasgow group acknowledges the financial support of the University of Glasgow. GEO acknowledges the financial support of the Particle Physics and Astronomy Research Council (PPARC), the Bundesministerium für Bildung und Forschung (BMBF) and the state of Lower Saxony. The LIGO Laboratory thanks the National Science Foundation for its support through the cooperative agreement PHY-9210038 and the award PHY-9801158.

\section{Appendix A}

We include here a brief discussion of the modelling tools used to produce the thermal noise and isolation curves presented in section 4 . 
Appendix A.1. Thermal noise model

The thermal noise associated with the suspension system is calculated using the fluctuationdissipation theorem [28]. The calculations in the code are carried out in the following way. The pendulum dynamics are simulated by four point-like masses linked by springs for both horizontal and vertical degrees of freedom, with no coupling between the orthogonal degrees of freedom. Suitable values to be used as input for the masses and other necessary parameters to calculate spring constants have previously been established using the MATLAB model of the quadruple pendulum, discussed in the following section. The first three spring stages consist of maraging steel blades in series with steel wires, and the final (lowest) stage consists of silica fibres. The horizontal and vertical transfer functions are calculated separately and then combined to get the effective overall horizontal function, assuming a cross-coupling of the vertical into the horizontal dimension of $0.1 \%$. This is a figure we have used in GEO [5] as a conservative estimate for cross-coupling, and is larger than the purely geometric effect due the curvature of the Earth over the $4 \mathrm{~km}$ arms of LIGO. Dissipation in the pendulum is introduced via the imaginary part of the spring constants, and hence using the fluctuation-dissipation theorem, the resulting thermal noise at the mirror in the horizontal direction is obtained.

Spring constants of the steel stages have been treated differently from the silica stage. For the steel the loss is included, with a dilution factor as appropriate, by including an imaginary term in the spring constant. For silica, the spring constants have been worked out from the solution of the beam equation, following the method used in Gonzalez and Saulson [29], in which case the imaginary part is introduced into Young's modulus. As a consequence, the programme calculates the violin modes of the silica stage, but not of the steel stages.

Loss angles for the materials arise as the sum of three parts: bulk, surface and thermoelastic effects, including the new thermoelastic effect referred to in section 2, which is included where appropriate. The surface loss is estimated following the work by Gretarsson and Harry [30], which indicated that there is an energy loss proportional to the surface to volume ratio for silica which dominates the bulk dissipation. For steel, however, the bulk loss dominates. The thermoelastic loss term has been considered in the pendulum motion of all four stages and in the vertical motion of the three steel stages in which the restoring force dominantly arises from the bending of the blades.

\section{Appendix A.2. MATLAB model for isolation and control}

The MATLAB model (recently extended to work in Simulink) consists at present of four uncoupled sets of dynamical equations, corresponding to vertical motion, yaw, longitudinal and pitch (together) and transverse and roll (together). To first order these motions are uncoupled in the GEO design. Forces due to gravity and extension of wires are included, but not due to the bending of wires. Cantilevers with wire(s) attached are approximated by taking the series sum of the spring constants of wire(s) and cantilever, noting that this sum is dominated by the softer cantilever blade. The model makes use of presumed symmetries in the design. With the crossed blades in the LIGO design, there will be some coupling between the longitudinal/pitch and transverse/roll modes. As yet the model does not incorporate this coupling. However, it is not expected to significantly affect either the isolation or the damping properties of the pendulum. In addition, the model does not yet take account of the twisting of the blade tips which will occur as the pendulum moves in the various pitch modes. Experimentally, we have seen that this effect slightly lowers the pitch modes. However, again the isolation and damping should not be significantly affected.

It should also be noted that the violin modes and the internal modes of the blades are not included in this MATLAB model. The violin modes of the final stage are, however, included in 
the thermal noise model, and they can be seen in the thermal noise curves shown in section 4 . The expected frequencies of the internal modes of the blades can be calculated from the dimensions of the blades, and are specific to each design of blade. Examples of their typical values were given in section 5 .

\section{References}

[1] Barish B C 1997 Gravitational Wave Detection ed K Tsubono, M-K Fujimoto and K Kurodo (Tokyo: Universal Academy) pp 155-61

[2] Coyne D 1996 IEEE Aerospace Applications Conf. Proc. vol 4 pp 31-61

[3] Hazel J, Kawamura S and Raab F 1996 LIGO Document T960074-07-D (December)

[4] Lück H et al 2000 Proc. 3rd Edoardo Amaldi Conf. on Gravitational Waves (Pasadena 1999) (American Institute of Physics) pp 119-27

[5] Plissi M V, Strain K A, Torrie C I, Robertson N A, Killbourn S, Rowan S, Twyford S M, Ward H, Skeldon K D and Hough J 1998 Rev. Sci. Instrum. $693055-61$

[6] Plissi M V, Torrie C I, Husman M E, Robertson N A, Strain K A, Ward H, Lück H and Hough J 2000 Rev. Sci. Instrum. $712539-45$

[7] Husman M E, Torrie C I, Plissi M V, Robertson N A, Strain K A and Hough J 2000 Rev. Sci. Instrum. 71 2546-51

[8] Rowan S, Twyford S M, Hough J, Gwo D-H and Route R 1998 Phys. Lett. A 246 471-8

[9] Cagnoli G, Gammaitoni L, Hough J, Kovalik J, McIntosh S, Punturo M and Rowan S 2000 Phys. Rev. Lett. 85 2442-5

[10] Robertson N A et al 2000 Proc. 3rd Edoardo Amaldi Conf. on Gravitational Waves (Pasadena 1999) (American Institute of Physics) pp 313-9

[11] Gustafson E, Shoemaker D, Strain K and Weiss R 1999 LIGO Report T990089-00-D

[12] Saulson P R 1990 Phys. Rev. D 422437

[13] Logan J E, Hough J, Robertson N A, Danzmann K and Hutchins R 1996 Proc. 7th Marcel Grossman Meeting, (Stanford, USA: World Scientific) pp 1410-2

[14] Rowan S, Cagnoli G, McIntosh S, Hough J, Sneddon P, Fejer M M, Gustafson E and Route R 1999 Proc. 2nd TAMA Int. Workshop on Gravitational Wave Detection (Tokyo, 1999) Gravitational Wave Detection II, 2000 Frontiers Science Series No 32 (Tokyo: Universal Academy) pp 203-15

[15] Gretarsson A M, Harry G M, Penn S D, Saulson P R, Startin W J, Rowan S, Cagnoli G and Hough J 2000 Phys. Lett. A 270 108-14

[16] Nowick A S and Berry B S 1972 Anelastic Relaxation in Crystalline Solids (New York: Academic)

[17] Rowan S, Hutchins R, McLaren A, Robertson N A, Twyford S M and Hough J 1997 Phys. Lett. A 227 153-8

[18] Cagnoli G and Willems P 2002 Phys. Rev. B 65174111

[19] Willems P 2002 Phys. Lett. A at press

[20] Gammaitoni L, Kovalik J, Marchesoni F, Punturo M and Cagnoli G 2000 Proc. 3rd Edoardo Amaldi Conf. on Gravitational Waves (Pasadena 1999) (American Institute of Physics) pp 162-72

[21] Torrie C I 1999 PhD Thesis University of Glasgow

[22] Brillet A (VIRGO Collaboration) 1997 Gravitational Wave Detection ed K Tsubono, M-K Fujimoto and K Kurodo (Tokyo: Universal Academy) pp 163-73

[23] Shoemaker D, Schilling R, Schnupp L, Winkler W, Maischberger K and Rüdiger A 1988 Phys. Rev. D 38 423-32

[24] Robertson D I, Morrison E, Hough J, Killbourn S, Meers B J, Newton G P, Robertson N A, Strain K A and Ward H 1995 Rev. Sci. Instrum. 664447

[25] Fritschel P, Bork R, Gonzalez G, Mavalvala N, Ouimette D, Rong H, Sigg D and Zucker M 2001 Appl. Opt. 40 4988-8

[26] Ando M and Tsubono K (TAMA Collaboration) 2000 Proc. 3rd Edoardo Amaldi Conference on Gravitational Waves (Pasadena 1999) (American Institute of Physics) pp 128-39

[27] Abbott R et al 2002 Class. Quantum Grav. 19 1591-7

[28] Callen H B and Welton T A 1951 Phys. Rev. 83 34-40

[29] Gonzalez G I and Saulson P R 1994 J. Acoust. Soc. Am. 96 207-12

[30] Gretarsson A M and Harry G M 1999 Rev. Sci. Instrum. 70 4081-7 